\title{
LAURA ARAGONE
}

\section{Problems of economic system optimization with quadratic criteria and monotone controls. Some algorithms for its numerical solution}

Revue française d'automatique, d'informatique et de recherche opérationnelle. Recherche opérationnelle, tome 27, n 1 (1993), p. $23-43$.

<http://www.numdam.org/item?id=RO_1993_27_1_23_0>

(C) AFCET, 1993, tous droits réservés.

L'accès aux archives de la revue « Revue française d'automatique, d'informatique et de recherche opérationnelle. Recherche opérationnelle » implique l'accord avec les conditions générales d'utilisation (http://www.numdam.org/ legal.php). Toute utilisation commerciale ou impression systématique est constitutive d'une infraction pénale. Toute copie ou impression de ce fichier doit contenir la présente mention de copyright.

\section{Numdam}




\title{
PROBLEMS OF ECONOMIC SYSTEM OPTIMIZATION WITH QUADRATIC CRITERIA AND MONOTONE CONTROLS SOME ALGORITHMS FOR ITS NUMERICAL SOLUTION (*)
}

\author{
by Laura Aragone ( ${ }^{1}$ ) \\ Communicated by E. GeLENBE
}

\begin{abstract}
In this paper some numerical algorithms are presented to solve the Hamilton-JacobiBellman equation associated to the optimal cost function corresponding to control problems of economic systems which involve the exploitation of non-renewable resources, i. e., the controls used are monotone (non-increasing or non-decreasing), and they may also be discontinuous. An almost complete study of this problem and of the theoretical characterization of its solution has been done in [2]. In that paper the solution of the problems is reduced to the treatment of an elliptic quasi-variational inequality in the interval $[0, T]$. In this paper an extension of the methodology introduced in [4] to the analysis and numerical solution of this inequality is done. We give three numerical algorithms, convergence properties of the discretization procedure is proven and comparative computational results obtained when solving the example introduced in [2] with the methods developed are shown.
\end{abstract}

Keywords: Quasi-variational inequalities; monotone controls; numerical solution; convergence rate; non-renewable resources; economic systems; quadratic criteria.

Résumé. - On présente ici quelques algorithmes numériques pour résoudre l'équation de Hamilton-Jacobi-Bellman associée aux problèmes d'optimisation de systèmes économiques où apparaissent des ressources non-renouvelables. Les contrôles sont monotones et ils peuvent être discontinus.

Une étude presque complète de ce problème avec la caractérisation théorique de la solution a été faite dans [2]. Là, la solution a été réduite au traitement d'une inéquation quasi-variationnelle elliptique dans l'intervalle $[0, T]$.

On fait ici une extension de la méthodologie introduite dans [4] à l'analyse et à la solution numérique de cette inéquation. On présente trois algorithmes numériques. On démontre la convergence de la discrétisation employée. Des résultats numériques, avec les temps de calcul sont présentés.

Mots clés : Inéquation quasi-variationnelle ; contrôle monotone ; solution numérique ; vitesse de convergence ; ressources non-renouvelables ; systèmes économiques ; critère quadratique.

(*) Received April 1992.

( ${ }^{1}$ Instituto de Matemática Beppo Levi, Facultad de Ciencas Exactas, Ingeniería y Agrimensura, Universidad Nacional de Rosario, Avenida Pellegrini 250, (2000) Rosario, Argentina.

Recherche opérationnelle/Operations Research, 0399-0559/93/01 23 21/\$ 4.10

(c) AFCET-Gauthier-Villars 


\section{INTRODUCTION}

An optimal control problem of a dynamic one-dimensional system described by an ordinary differential equation is considered. The time interval considered is finite; the functional to be optimized is quadratic and takes the following explicit expression:

$$
\min _{p(\cdot)} \int_{0}^{T} e^{-\alpha s}\left\{L[y(s)-f(s)]^{2}+M\left[p(s)-f^{\prime}(s)\right]^{2}\right\} d s
$$

being

$$
\frac{d y}{d t}=p(t)
$$

$p$ is the control variable and it belongs to $\mathcal{R}^{+}=[0, \infty)$.

$\alpha$ is the discount coefficient, $L$ and $M$ the weighting factors, and $f$ is the trajectory to pursue.

The minimization problem studied has free initial conditions; therefore, what we must really seek is the minimum with respect to $y(0), p($.$) .$

Consequently, we have that $y$ is a non-decreasing variable; that is why, this type of problems receive the name of monotone controls since we may consider $y$ as the control variable.

This type of problems appear in economic issues such as the control of monetary policy, where $p$ represents the injection of money and $y$ is the monetary stock. Similar problems occur when we consider the control of the exploitation of exhaustible resources, such as pumping oil, mining ore, harvesting fish or trees, etc. In [2] has been presented the characterization of the solution of this problem using the methodology of variational equations in Sobolev spaces

By defining

$$
Y(t)=\int_{0}^{t} y(s) d s \quad F(t)=\int_{0}^{t} f(s) d s
$$

the optimization problem may be expressed in terms of $Y(\cdot)$ and consequently, the following differential equation of optimality for $Y$ is obtained

$$
Y^{\prime \prime}+\left(Y-F+F^{\prime \prime}\right)^{-}=0 \quad \text { in }(0, \mathrm{~T})
$$

with boundary conditions

$$
\begin{gathered}
Y(0)=0 \\
Y(T)=F(T)
\end{gathered}
$$

(where $w^{-}$denotes the negative part of the scalar $w$ ). 
This conditions may also be obtained directly by applying the Pontryaguin's maximum principle. In particular (5) is a direct consequence of considering the optimization with free initial conditions for $y(0)$.

The solution of (4) has the following minimality property:

THEOREM 1.1:

$$
Y \leq u \text { in }[0, T], \quad \forall u \in U
$$

being

$$
\begin{aligned}
U=\left\{u \in H^{2}[0, T] / u^{\prime \prime} \leq 0, u^{\prime \prime}-\right. & \\
& \leq F^{\prime \prime}-F, u(0) \geq 0, u(T) \geq F(T)
\end{aligned}
$$

(The proof is included in [2].)

Set $U$ is called the set of supersolutions. By virtue of the characterization given by theorem 1.1 for the element $Y$, the original problem is reduced to determine the minimum element of $U$. To find it numerically, a discretization procedure is developed by applying the methodology introduced in [5]. The method is based on the use of linear finite elements and on the use of a suitable discretization of the conditions which appear in (8).

\section{THE DISCRETIZED PROBLEM AND ITS SOLUTIONS}

We use an external approximation of the space $H^{2}[0, T]$ given by linear finite elements (we denote with $W^{h}$ the set of those elements, which belong to $\left.H^{1}[0, T]\right)$. Consequently, these approximating functions are determined by the values they assume in the nodes of the discretization. In this onedimensional case those values will be (for a uniform partition of $[0, T]$ ):

$$
\Omega^{h}=\left\{t_{i} / t_{i}=i h ; i=0,1, \ldots, n\right\} \text { a partition of } \Omega, \quad h=\frac{T}{n}
$$

DeFINITION 2.1: Let $u^{h} \in W^{h}$

We define $S \subset N^{h}=\{0,1,2, \ldots, n\}$

- $i^{-}(S)=\max (j / j<i, j \in S)$

- $i^{+}(S)=\min (j / j>i, j \in S)$

- $Y L\left(u^{h}, S, i\right)$

$$
=\left(\left(i^{+}-i\right) u^{h}\left(t_{i^{-}}\right)+\left(i-i^{-}\right) u^{h}\left(t_{i^{+}}\right)\right) /\left(i^{+}-i^{-}\right)
$$


- $D^{2}\left(u^{h}, S, i\right)=\left(\frac{u^{h}\left(t_{i^{+}}\right)-u^{h}\left(t_{i}\right)}{\left(t_{i^{+}}-t_{i}\right)}-\frac{u^{h}\left(t_{i}\right)-u^{h}\left(t_{i^{-}}\right)}{\left(t_{i}-t_{i^{-}}\right)}\right) / h$

- $G\left(u^{h}, S, i\right)=\left(\left(D^{2}\left(F, N^{h}, i\right)\right.\right.$

$$
\begin{aligned}
& \left.-F\left(t_{i}\right)\right)\left(h\left(t_{i}-t_{i^{-}}\right)\left(t_{i^{+}}-t_{i}\right)\right)+\left(t_{i^{+}}-t_{i}\right) u^{h}\left(t_{i^{-}}\right) \\
& \left.+\left(t_{i}-t_{i^{-}}\right) u^{h}\left(t_{i^{+}}\right)\right) /\left(h\left(t_{i}-t_{i^{-}}\right)\left(t_{i^{+}}-t_{i}\right)+\left(t_{i^{+}}-t_{i^{-}}\right)\right)
\end{aligned}
$$

The discretization of the restrictions which define $U$ determine a set of discrete supersolutions $U^{h}$ which has the following form:

$$
\begin{gathered}
U^{h}=\left\{u^{h} \in W^{h} / u^{h} \text { verifies }(15)-(18)\right\} \\
u^{h}\left(t_{0}\right) \geq 0 \\
u^{h}\left(t_{n}\right) \geq F(T) \\
D^{2}\left(u^{h}, N^{h}, i\right) \leq 0, \quad i=1, \ldots, n-1 \\
u^{h}\left(t_{i}\right) \geq G\left(u^{h}, N^{h}, i\right), \quad i=1, \ldots, n-1
\end{gathered}
$$

Function $Y^{h}$, minimum element of $U^{h}$ is characterized by the conditions:

$$
\begin{gathered}
Y^{h}\left(t_{0}\right)=0 \\
Y^{h}\left(t_{n}\right)=F(T) \\
Y^{h}\left(t_{i}\right)=\max \left\{Y L\left(Y^{h}, N^{h}, i\right), G\left(Y^{h}, N^{h}, i\right)\right\}, \\
i=1, \ldots, n-1
\end{gathered}
$$

Operator $P: W^{h} \rightarrow W^{h}$ is introduced in the following way

$$
\begin{gathered}
\left(P w^{h}\right)\left(t_{0}\right)=0 \\
\left(P w^{h}\right)\left(t_{n}\right)=F(T) \\
P w^{h}\left(t_{i}\right)=\max \left\{Y L\left(w^{h}, N^{h}, i\right), G\left(w^{h}, N^{h}, i\right)\right\}, \\
i=1, \ldots, n-1
\end{gathered}
$$

This operator is monotone and increasing and leaves set $U^{h}$ invariant. Moreover, it can be proved that although $P$ is not contractive, $P^{[n / 2]}$ is contractive and consequently, $P$ has only one fixed point, which coincides with the unique fixed point of $P^{[n / 2]}$. That is, if we denote with $\tilde{Y}^{h}$ the unique solution of the equation:

$$
\tilde{Y}^{h}=P^{[n / 2]} \tilde{Y}^{h}
$$


it also results $\tilde{Y}^{h}=Y^{h}$ and therefore, it is also a solution of equation

$$
Y^{h}=P Y^{h}
$$

In consequence, the solution of the discretized problem, i.e. the minimum element of $U^{h}$, is characterized as the solution of equation (26). At the same time, operator $P$ allows the definition of an algorithm to calculate $Y^{h}$.

\section{Discretized problem:}

$$
\text { Find } Y^{h} \in \mathcal{R}^{n}, \text { such that } P Y^{h}=Y^{h}
$$

\section{CONVERGENCE OF APPROXIMATE SOLUTIONS}

The functions $Y^{h}$, solutions of the discrete problem, converge in a uniform way to function $\bar{Y}$ which is solution of (4), when $n \rightarrow \infty(h \rightarrow 0)$.

In effect, by virtue of the type of discretization used, we have that a discrete maximum principle holds, which in turns, implies the uniform convergence of the approximations (see [3]).

Let

$Y^{h}$ the solution of the discretized problem (i.e. $P Y^{h}=Y^{h}$ )

$\bar{Y}$ the analytical solution

$\bar{Y}^{h}$ the interpolation of the analytical solution (i.e. $\bar{Y}^{h}\left(t_{i}\right)=\bar{Y}\left(t_{i}\right)$ )

Theorem 3.1: If $F \in H^{3}[0, T]$ then

$$
\left\|Y^{h}-\bar{Y}\right\| \leq K \sqrt{h}
$$

The proof of (27) is a direct consequence of remark 3.1 and of lemmas 3.1 and 3.3.

Proposition 3.1: If $a \in H^{3}[0, T]$ and $a^{h}$ is its linear interpolation then $\exists C \geq 0$ such that

$$
\left|D^{2}\left(a^{h}, N^{h}, i\right)-a^{\prime \prime}\left(t_{i}\right)\right| \leq C \sqrt{h}, \quad \forall i=1, \ldots, n-1
$$


Proof: If $a \in H^{3}[0, T]$, we have the following Taylor's expansion:

$$
\begin{aligned}
& a(t+h)=a(t)+a^{\prime}(t) h+a^{\prime \prime}(t) \frac{h^{2}}{2}+\frac{1}{2 !} \int_{0}^{h} \tau^{2} a^{\prime \prime \prime}(\tau+t) d \tau \\
& a(t-h)=a(t)-a^{\prime}(t) h+a^{\prime \prime}(t) \frac{h^{2}}{2}+\frac{1}{2 !} \int_{0}^{-h} \tau^{2} a^{\prime \prime \prime}(\tau+t) d \tau
\end{aligned}
$$

Thus

$$
\begin{aligned}
& D^{2}\left(a^{h}, N^{h}, i\right)=\frac{a\left(t_{i^{+}}\right)+a\left(t_{i^{-}}\right)-2}{h^{2}} \\
&=a^{\prime \prime}\left(t_{i}\right)+\frac{1}{2 h^{2}} \int_{-h}^{h} \tau^{2} a^{\prime \prime \prime}\left(\tau+t_{i}\right) d \tau
\end{aligned}
$$

By the Hölder inequality and considering that $a^{\prime \prime \prime} \in L^{2}[0, T]$

$$
\begin{aligned}
\left|\int_{-h}^{h} \tau^{2} a^{\prime \prime \prime}\left(\tau+t_{i}\right) d \tau\right| \leq\left(\int _ { - h } ^ { h } \left[a^{\prime \prime \prime}(\tau\right.\right. & \left.\left.\left.+t_{i}\right)\right]^{2} d \tau\right)^{1 / 2} \\
& \times\left(\int_{-h}^{h} \tau^{4} d \tau\right)^{1 / 2} \leq 2\left\|a^{\prime \prime \prime}\right\|_{L^{2}} h^{5 / 2}
\end{aligned}
$$

In consequence

$$
\left|D^{2}\left(a^{h}, N^{h}, i\right)-a^{\prime \prime}\left(t_{i}\right)\right| \leq C \sqrt{h}
$$

Remark 3.1: In [2], the following results is proved: if $F \in H^{3}[0, T]$ then $\bar{Y} \in H^{3}[0, T]$.

In consequence, by virtue of proposition 3.1, for $\bar{Y}$ we have:

$$
\left|D^{2}\left(\bar{Y}^{h}, N^{h}, i\right)-\bar{Y}^{\prime \prime}\left(t_{i}\right)\right| \leq C \sqrt{h}, \quad i=1, \ldots, n-1
$$

LEMMA 3.1:

$$
Y^{h}\left(t_{i}\right)-\bar{Y}^{h}\left(t_{i}\right)<C h T^{2} / 4, \quad \forall t_{i} \in N^{h}
$$

Proof: In a first place, we will prove that $\exists \phi^{h} / \bar{Y}^{h}+\phi^{h}$ is a supersolution, i.e. we must prove that $\bar{Y}^{h}+\phi^{h}=u^{h}$ verifies (15-18).

Let $\phi^{h}$ be the linear interpolation of the concave function

$$
\phi(t)=C \sqrt{h} t(T-t)
$$

then, it results

$$
\phi^{\prime \prime}(t)-\phi(t) \leq-2 C \sqrt{h}, \quad t \in[0, T]
$$

[note that $D^{2}\left(\phi^{h}, N^{h}, i\right)=\phi^{\prime \prime}\left(t_{i}\right)$ ], and obviously (15) and (16) are verified. 
Easily it can be proved that

$$
\begin{aligned}
D^{2}\left(\bar{Y}^{h}+\phi^{h}, N^{h}, i\right) & \leq \bar{Y}^{\prime \prime}\left(t_{i}\right)+C \sqrt{h}+\phi^{\prime \prime}\left(t_{i}\right) \leq-C \sqrt{h}, \\
i & =1, \ldots, n-1 .
\end{aligned}
$$

To prove (18), we consider that, by virtue of (28):

$$
\begin{aligned}
D^{2}\left(\bar{Y}^{h},\right. & \left.N^{h}, i\right)-\bar{Y}^{h}\left(t_{i}\right)-D^{2}\left(F, N^{h}, i\right)+F\left(t_{i}\right) \\
& \leq \bar{Y}^{\prime \prime}\left(t_{i}\right)+C \sqrt{h}-\bar{Y}\left(t_{i}\right)-\left(F^{\prime \prime}\left(t_{i}\right)-C \sqrt{h}\right)+F\left(t_{i}\right) \leq 2 C \sqrt{h} .
\end{aligned}
$$

Then

$$
D^{2}\left(\bar{Y}^{h}+\phi^{h}, N^{h}, i\right)-\left(\bar{Y}^{h}+\phi^{h}\right)\left(t_{i}\right)-D^{2}\left(F, N^{h}, i\right)+F\left(t_{i}\right) \leq 0
$$

Finally, $\bar{Y}^{h}+\phi^{h}$ is a supersolution and as $Y^{h}$ is the minimum element of the set $U^{h}$ of supersolutions, it results

$$
Y^{h}\left(t_{i}\right) \leq \bar{Y}^{h}\left(t_{i}\right)+\phi^{h}\left(t_{i}\right)
$$

and in consequence (29) holds.

LemMa 3.2: Let $g^{h}$ be a finite element defined in $\Omega^{h}$, such that

$$
\begin{aligned}
& g^{h}(0) \geq 0 \\
& g^{h}(T) \geq 0
\end{aligned}
$$

and $\forall t_{i} \in \Omega^{h}$ at least one of the following inequations is verified

$$
\begin{gathered}
D^{2}\left(g^{h}, N^{h}, i\right)<0 \\
D^{2}\left(g^{h}, N^{h}, i\right)-g^{h}\left(t_{i}\right) \leq 0
\end{gathered}
$$

then

$$
g^{h}\left(t_{i}\right) \geq 0, \quad \forall t_{i} \in \Omega^{h}
$$


Proof: If we suppose that (32) is not valid, i.e. there exists a negative minimum; then there would exists $t_{\hat{\imath}}$ such that

$$
\begin{gathered}
g^{h}\left(t_{\hat{\imath}}\right)<0 \\
g^{h}\left(t_{\hat{\imath}}\right) \leq g^{h}\left(t_{\hat{\imath}+1}\right) \\
g^{h}\left(t_{\hat{\iota}}\right) \leq g^{h}\left(t_{\hat{\imath}+1}\right)
\end{gathered}
$$

which implies

$$
D^{2}\left(g^{h}, N^{h}, \hat{\iota}\right)=\frac{g^{h}\left(t_{\hat{\imath}-1}\right)+g^{h}\left(t_{\hat{\imath}+1}\right)-2 g^{h}\left(t_{\hat{\iota}}\right)}{h^{2}} \geq 0
$$

Therefore

$$
D^{2}\left(g^{h}, N^{h}, \hat{\iota}\right)-g^{h}\left(t_{\hat{\iota}}\right)>0,
$$

which contradicts the hypothesis.

LEMMA 3.3:

$$
Y^{h}\left(t_{i}\right)-\bar{Y}^{h}\left(t_{i}\right) \geq-\frac{C}{4} T^{2} \sqrt{h}, \quad \forall t_{i} \in N^{h}
$$

Proof: We will prove that $g^{h}=Y^{h}-\bar{Y}^{h}+\phi^{h}$ satisfies conditions (30) and (31), with

$$
\phi^{h}\left(t_{i}\right)=C \sqrt{h} t_{i}\left(T-t_{i}\right)
$$

Let we define

$$
\begin{gathered}
\bar{C}_{1}^{h}=\left\{t_{i} \in \Omega^{h} / \bar{Y}^{\prime \prime}\left(t_{i}\right)=0\right\} \\
\bar{C}_{2}^{h}=\left\{t_{i} \in \Omega^{h} / \bar{Y}^{\prime \prime}\left(t_{i}\right)-\bar{Y}\left(t_{i}\right)+F^{\prime \prime}\left(t_{i}\right)-F\left(t_{i}\right)=0\right\}
\end{gathered}
$$

If $t_{i} \in \bar{C}_{1}^{h}$, we have

$$
\bar{Y}^{\prime \prime}\left(t_{i}\right)=0
$$

therefore, by remark 3.1

$$
D^{2}\left(\bar{Y}^{h}, N^{h}, i\right) \geq-C \sqrt{h}
$$

by (21)

$$
D^{2}\left(Y^{h}, N^{h}, i\right) \leq 0
$$


and, by the definition of $\phi^{h}$

$$
D^{2}\left(\phi^{h}, N^{h}, i\right)=-2 C \sqrt{h} .
$$

By virtue of these inequalities, we have

$$
D^{2}\left(g, N^{h}, i\right)<0
$$

If $t_{i} \in \bar{C}_{2}^{h}$, we have $\bar{Y}^{\prime \prime}\left(t_{i}\right)-\bar{Y}\left(t_{i}\right)-F^{\prime \prime}\left(t_{i}\right)+F\left(t_{i}\right)=0$ then

$$
\begin{aligned}
D^{2}\left(\bar{Y}^{h},\right. & \left.N^{h}, i\right)-\bar{Y}^{h}\left(t_{i}\right)-D^{2}\left(F, N^{h}, i\right)+F\left(t_{i}\right) \\
& \geq \bar{Y}^{\prime \prime}\left(t_{i}\right)-C \sqrt{h}-\bar{Y}\left(t_{i}\right)-\left(F^{\prime \prime}\left(t_{i}\right)+C \sqrt{h}\right)+F\left(t_{i}\right) \geq-2 C \sqrt{h}
\end{aligned}
$$

having in mind that

$$
D^{2}\left(Y^{h}, N^{h}, i\right)-Y^{h}\left(t_{i}\right)-D^{2}\left(F, N^{h}, i\right)+F\left(t_{i}\right) \leq 0
$$

and

$$
D^{2}\left(\phi^{h}, N^{h}, i\right)-\phi^{h}\left(t_{i}\right)=-2 C h^{1 / 2}-C h^{1 / 2} t_{i}\left(T-t_{i}\right) \leq-2 C \sqrt{h}
$$

results

$$
D^{2}\left(g, N^{h}, i\right)-g\left(t_{i}\right) \leq 0
$$

and due to Lemma 3.2

$$
g\left(t_{i}\right) \geq 0
$$

which implies

$$
Y^{h}\left(t_{i}\right)-\bar{Y}^{h}\left(t_{i}\right)+\phi^{h}\left(t_{i}\right) \geq 0
$$

That is,

$$
Y^{h}\left(t_{i}\right)-\bar{Y}^{h}\left(t_{i}\right) \geq-\phi^{h}\left(t_{i}\right)=-C h^{1 / 2} t_{i}\left(T-t_{i}\right) \geq-C \sqrt{h} T^{2} / 4 .
$$




\section{NUMERICAL SOLUTION OF THE DISCRETIZED PROBLEM}

\section{First algorithm}

The proof of the fixed point theorem hints us the following algorithm to compute $Y^{h}$.

\section{Algorithm A0}

Step 0: Compute $Y_{0} \in W^{h}$, set $\nu=0$.

Step 1: Set $Y_{\nu+1}=P_{h} Y_{\nu}$.

Step 2: If $Y_{\nu}=Y_{\nu+1}$, go to step 3; else, $\nu=\nu+1$ and go to step 1.

Step 3: Set $Y^{h}=Y_{\nu}$ and stop.

This algorithm finishes in a finite number of steps or it generates a sequence of elements $Y_{v}$ which converges to $Y^{h}$; in addition the following inequality is verified:

$$
\left\|Y_{\nu}-Y^{h}\right\| \leq(\tilde{\beta})^{\tilde{\nu}}\left\|Y_{0}-Y^{h}\right\|
$$

being $\tilde{\beta}<1$ and $\bar{\nu}=\operatorname{int}(\nu / n)$.

It can also be observed, by virtue of the properties of $P_{h}$, that if a initial point $Y_{0} \in U^{h}$ is chosen, the sequence $Y_{\nu}$ verifies:

$$
\begin{gathered}
Y_{\nu} \in U^{h} \\
Y_{\nu} \geq Y_{\nu+1} \geq Y^{h}
\end{gathered}
$$

\section{AN ACCELERATED ALGORITHM}

\subsection{Foundations of the method}

When applying algorithm A0, it can generally be observed that after few iterations, the sets of nodes where conditions (17) and (18) are respectively satisfied with the sign=remains invariant, and the algorithm simply tries to solve in an iterative way the corresponding associated linear system. The following accelerated algorithm makes use of the observation of this phenomenon. Also, it uses the fact that in the regions where (17) is satisfied with the $\operatorname{sign}=$, the solution function is obviously a linear function of the values at the extreme points of these regions. 


\subsection{Detailed description of the algorithm}

Defintrion 5.2.1: Let $Y \in W^{h}$, we define TEST(Y, S)=TRUE if either $\operatorname{Card}(\mathrm{S})=2$ or

$\operatorname{Card}(\mathrm{S}) \geq 3$

$D^{2}(Y, S, i) \leq 0, \quad \forall i \in S / i=1, \ldots, n-1$

$D^{2}(Y, S, i)-Y\left(t_{i}\right) \leq D^{2}\left(F, N^{h}, i\right)-F\left(t_{i}\right), \quad \forall i \in S / i=1, \ldots, n-1$

\section{Algorithm A1}

Step 1: Set

$$
\begin{aligned}
& Y_{1,0}\left(t_{i}\right)=F\left(t_{i}\right), \quad i=0,1, \ldots, n \\
& m=0, \quad \nu=1 \\
& S_{1,0}=N^{h}
\end{aligned}
$$

Step 2: Set $j=1$

Step 3: Set $m=m+1$

$$
\begin{aligned}
& \text { If } D^{2}\left(Y_{\nu, m-1}, S_{\nu, m-1}, j\right) \geq 0 \text {, then } \\
& \text { If }-Y L\left(Y_{\nu, m-1}, S_{\nu, m-1}, j\right) \leq D^{2}\left(F, N^{h}, j\right)-F\left(t_{j}\right) \text {, then } \\
& S_{\nu, m}=S_{\nu, m-1}-\{j\} \\
& j_{\nu, m}=j \\
& Y_{\nu, m}\left(t_{i}\right)=Y_{\nu, m-1}\left(t_{i}\right), \quad \forall i \neq j_{\nu, m} \\
& Y_{\nu, m}\left(t_{j, m}\right)=Y L\left(Y_{\nu, m-1}, S_{\nu, m-1}, j_{\nu, m}\right) \\
& j=j-\left(S_{\nu, m}\right) \\
& \text { If } j \neq 0, \text { then go to step 3; else, go to step } 5 \\
& \text { else, go to step 4 } \\
& \text { else; go to step 4 }
\end{aligned}
$$

Step 4: Set

$$
\begin{aligned}
& S_{\nu, m}=S_{\nu, m-1} \\
& j_{\nu, m}=j \\
& Y_{\nu, m}\left(t_{i}\right)=Y_{\nu, m-1}\left(t_{i}\right), \quad \forall i \neq j_{\nu, m} \\
& Y_{\nu, m}\left(t_{j_{\nu, m}}\right)=G\left(Y_{\nu, m-1}, S_{\nu, m-1}, j_{\nu, m}\right)
\end{aligned}
$$


Step 5: Set $j=j^{+}\left(S_{\nu, m}\right)$

If $j \leq n-1$, then go to step 3; else, $m_{\nu}=m$ and go to step 6

Step 6: Set

$$
\begin{gathered}
\nu=\nu+1, S_{\nu, 0}=S_{\nu-1, m} \\
m=0 \\
Y_{\nu, m}\left(t_{0}\right)=F\left(t_{0}\right) \\
Y_{\nu, m}\left(t_{n}\right)=F\left(t_{n}\right)
\end{gathered}
$$

- If $\operatorname{Card}\left(S_{\nu, m}\right) \geq 3$ solve the linear system:

$$
\begin{aligned}
D^{2}\left(Y_{\nu, m}, S_{\nu, m}, i\right)- & Y_{\nu, m}\left(t_{i}\right) \\
& =D^{2}\left(F, N^{h}, i\right)-F\left(t_{i}\right), \quad \forall i \in S_{\nu, 0} / 0<i<n
\end{aligned}
$$

- $Y_{\nu, m}\left(t_{j}\right)=Y L\left(Y_{\nu, m}, S_{\nu, m}, j\right), \quad \forall j \notin S_{\nu, 0}$

Step 7: If $\operatorname{TEST}\left(Y_{\nu, m}, S_{\nu, m}\right)=$ TRUE stop and $Y_{\nu, m}$ is the solution else go to step 2 .

\subsection{Properties}

The special properties of this algorithm are described in detail in the following paragraphs.

Remark 5.1:

$$
\begin{aligned}
& D^{2}\left(Y_{\nu, m}, S_{\nu, m}, i\right)=D^{2}\left(Y_{\nu, m}, N^{h}, i\right) \\
& \forall i \in S_{\nu, m} \backslash\{0, n\}, \quad \forall m, \quad \forall \nu \\
& D^{2}\left(Y_{\nu, m}, S_{\nu, m}, i\right) \\
& =\frac{Y_{\nu, m}\left(t_{i^{+}}\right)-Y_{\nu, m}\left(t_{i}\right)}{t_{i^{+}}-t_{i}}-\frac{Y_{\nu, m}\left(t_{i}\right)-Y_{\nu, m}\left(t_{i^{-}}\right)}{t_{i}-t_{i^{-}}} \\
& =\frac{Y_{\nu, m}\left(t_{i^{+}}\right)-Y_{\nu, m}\left(t_{i}\right)}{t_{i^{+}}-t_{i}}-\frac{Y_{\nu, m}\left(t_{i}\right)-Y_{\nu, m}\left(t_{i-1}\right)}{t_{i}-t_{i-1}} \\
& =D^{2}\left(Y_{\nu, m}, N^{h}, i\right)
\end{aligned}
$$

since $Y_{\nu, m}\left(t_{j}\right)$ is linear $\forall j \notin S_{\nu, m}$.

LemMa 5.1:

$$
\begin{gathered}
Y_{\nu, m}\left(t_{i}\right) \leq G\left(Y_{\nu, m}, S_{\nu, m}, i\right), \\
\forall i \in S_{\nu, m} \backslash\{0, n\}, \quad \forall \nu \geq 1, \quad \forall 0 \leq m \leq m_{\nu}
\end{gathered}
$$


Proof: It will be given by induction on $m$. So, for $m=0$, we have:

$$
\begin{aligned}
D^{2}\left(Y_{\nu, 0}, S_{\nu, 0}, i\right)-Y_{\nu, 0}\left(t_{i}\right)=D^{2}\left(F, N^{h}, i\right)-F\left(t_{i}\right) & \\
& \forall i \in S_{\nu, 0} / 0<i<n
\end{aligned}
$$

and this is equivalent to

$$
Y_{\nu, 0}\left(t_{i}\right)=G\left(Y_{\nu, 0}, S_{\nu, 0}, i\right), \quad \forall i \in S_{\nu, 0} i \neq(0, n), \quad \forall \nu \geq 1
$$

We suppose (34) is satisfied for $m-1 \geq 0$ and we will prove it for $m$.

The values of $Y_{\nu, m}\left(t_{i}\right)$ are identical to those of $Y_{\nu, m-1}\left(t_{i}\right)$ for all $i \in S_{\nu, m}, i \neq j_{\nu, m}$ then, due to definition 2.1, for $i<\left(j_{\nu, m}\right)^{-}$and for $i>\left(j_{\nu, m}\right)^{+}$, we have:

$$
\begin{aligned}
& Y_{\nu, m}\left(t_{i}\right)=Y_{\nu, m-1}\left(t_{i}\right) \leq G\left(Y_{\nu, m-1}, S_{\nu, m-1}, i\right) \\
& \quad=G\left(Y_{\nu, m}, S_{\nu, m-1}, i\right)=G\left(Y_{\nu, m}, S_{\nu, m}, i\right)
\end{aligned}
$$

since for these values of $i$

$$
i^{ \pm}\left(S_{\nu, m-1}\right)=i^{ \pm}\left(S_{\nu, m}\right)
$$

We are going now to show that

$$
Y_{\nu, m}\left(t_{j_{\nu, m}}\right) \geq Y_{\nu, m-1}\left(t_{j_{\nu, m}}\right)
$$

- If $j_{\nu, m} \in S_{\nu, m}$ we have by the induction hypothesis

$$
Y_{\nu, m}=G\left(Y_{\nu, m-1}, S_{\nu, m-1}, j_{\nu, m-1}\right) \geq Y_{\nu, m-1}\left(t_{j_{\nu, m}}\right)
$$

and so, (35) is valid.

- If $j_{\nu, m} \notin S_{\nu, m} \Rightarrow D^{2}\left(Y_{\nu, m-1}, S_{\nu, m-1}, j_{\nu, m}\right) \geq 0$ then

$$
Y_{\nu, m}\left(t_{j_{\nu, m}}\right)=Y L\left(Y_{\nu, m-1}, S_{\nu, m-1}, j_{\nu, m}\right)
$$

and so, (35) is valid.

Now we will prove that the thesis is satisfied for $\left(j_{\nu, m}\right)^{-}, j_{\nu, m},\left(j_{\nu, m}\right)^{+}$.

For $i=\left(j_{\nu, m}\right)^{-}$

$$
Y_{\nu, m}\left(t_{i}\right)=Y_{\nu, m-1}\left(t_{i}\right) \leq G\left(Y_{\nu, m-1}, S_{\nu, m-1}, i\right)
$$


- If $j_{\nu, m} \in S_{\nu, m}$ we have by virtue of (35)

$$
G\left(Y_{\nu, m-1}, S_{\nu, m-1}, i\right) \leq G\left(Y_{\nu, m}, S_{\nu, m}, i\right)
$$

- If $j_{\nu, m} \notin S_{\nu, m}$ we have

$$
\begin{gathered}
i^{+}\left(S_{\nu, m}\right)=\left(j_{\nu, m}\right)^{+}\left(S_{\nu, m-1}\right) \\
i^{+}\left(S_{\nu, m-1}\right)=j_{\nu, m} \\
i^{-}\left(S_{\nu, m-1}\right)=i^{-}\left(S_{\nu, m}\right)
\end{gathered}
$$

Note that proving the thesis is equivalent to prove that

$$
D^{2}\left(Y_{\nu, m}, S_{\nu, m}, i\right)-Y_{\nu, m}\left(t_{i}\right) \geq D^{2}\left(f, N^{h}, i\right)-f\left(t_{i}\right)
$$

which by the induction hypothesis is valid for $m-1$.

As

$$
Y_{\nu, m-1}\left(t_{i}\right)=Y_{\nu, m}\left(t_{i}\right)
$$

it will be enough to see that

$$
D^{2}\left(Y_{\nu, m-1}, S_{\nu, m-1}, i\right) \leq D^{2}\left(Y_{\nu, m}, S_{\nu, m}, i\right)
$$

Due to remark 5.1, the left side of this inequality becomes

$$
\begin{aligned}
& \frac{Y_{\nu, m-1}\left(t_{j}\right)-Y_{\nu, m-1}\left(t_{i}\right)}{t_{j}-t_{i}}-\frac{Y_{\nu, m-1}\left(t_{i}\right)-Y_{\nu, m-1}\left(t_{i^{-}}\right)}{t_{i^{-}}-t_{i^{-}}} \\
& =\frac{Y_{\nu, m-1}\left(t_{j}\right)-Y_{\nu, m}\left(t_{i}\right)}{t_{i^{+}}-t_{i}}-\frac{Y_{\nu, m}\left(t_{i}\right)-Y_{\nu, m}\left(t_{i^{-}}\right)}{t_{i^{-}}-t_{i^{-}}} \\
& \leq \frac{Y_{\nu, m}\left(t_{j}\right)-Y_{\nu, m}\left(t_{i}\right)}{t_{i^{+}}-t_{i}}-\frac{Y_{\nu, m}\left(t_{i}\right)-Y_{\nu, m}\left(t_{i^{-}}\right)}{t_{i}-t_{i^{-}}}=D^{2}\left(Y_{\nu, m}, S_{\nu, m}, i\right)
\end{aligned}
$$

In the same way for $i=J^{+}\left(S_{v, m}\right)$

Now, let $i=j_{\nu, m}$. We must deal only with the case $j_{\nu, m} \in S_{\nu, m}$. But in this case it is

$$
Y_{\nu, m}\left(t_{j_{\nu, m}}\right)=G\left(Y_{\nu, m}, S_{\nu, m}, j_{\nu, m}\right)
$$

LeMma 5.2: The algorithm is increasing, i.e.

$$
\begin{gathered}
Y_{\nu, m+1}\left(t_{i}\right) \geq Y_{\nu, m}\left(t_{i}\right), \quad \forall i \in N^{h}, \quad \forall \nu \geq 1, \quad \forall 0 \leq m<m_{\nu} \\
Y_{\nu+1,0}\left(t_{i}\right) \geq Y_{\nu, m_{\nu}}\left(t_{i}\right), \quad \forall i \in N^{h}, \quad \forall \nu \geq 1
\end{gathered}
$$


Proof: 1 . Since in the algorithm the values of $Y_{\nu, m}\left(t_{0}\right)$ and $Y_{\nu, m}\left(t_{n}\right)$ are not modified, (36) is trivially satisfied for all $v, m$.

2. We will prove that

$$
Y_{\nu, m+1}\left(t_{i}\right) \geq Y_{\nu, m}\left(t_{i}\right), \quad \forall 0<i<n, \quad \forall \nu \geq 1, \quad \forall 0 \leq m<m_{\nu}
$$

By construction of $Y_{\nu, m}$, we have

$$
Y_{\nu, m}\left(t_{i}\right)=Y_{\nu, m-1}\left(t_{i}\right), \quad \forall 0<i<n / i \neq j_{\nu, m}
$$

For $i=j_{\nu, m}$ we divide the analysis in two cases

- $j_{\nu, m} \notin S_{\nu, m}$

$$
Y_{\nu, m}\left(t_{j_{\nu, m}}\right)=Y L\left(Y_{\nu, m-1}, S_{\nu, m-1}, j_{\nu, m}\right)
$$

As it is evident that

$$
\begin{aligned}
D^{2}\left(Y_{\nu, m-1}, S_{\nu, m-1}, j\right) & \geq 0 \\
& \quad \text { implies } Y_{\nu, m-1}\left(t_{j_{\nu, m}}\right) \leq Y L\left(Y_{\nu, m-1}, S_{\nu, m-1}, j_{\nu, m}\right)
\end{aligned}
$$

then, we have

$$
Y_{\nu, m}\left(t_{j_{\nu, m}}\right) \geq Y_{\nu, m-1}\left(t_{j_{\nu, m}}\right)
$$

$\cdot j_{\nu, m} \in S_{\nu, m}$

$$
Y_{\nu, m}\left(t_{j_{\nu, m}}\right)=G\left(Y_{\nu, m-1}, S_{\nu, m-1}, j_{\nu, m}\right)
$$

but, due to (36)

$$
Y_{\nu, m-1}\left(t_{j_{\nu, m}}\right) \leq G\left(Y_{\nu, m-1}, S_{\nu, m-1}, j_{\nu, m}\right)
$$

therefore

$$
Y_{\nu, m}\left(t_{j_{\nu, m}}\right) \geq Y_{\nu, m-1}\left(t_{j_{\nu, m}}\right)
$$

3. We define the auxiliary function

$$
g\left(t_{i}\right)=Y_{\nu+1,0}\left(t_{i}\right)-Y_{\nu, m_{\nu}}\left(t_{i}\right)
$$

Then, by virtue of (36), remark 5.1 and the definition of step 6 , we have that

$$
\begin{gathered}
D^{2}\left(g, N^{h}, i\right)-g\left(t_{i}\right) \leq 0, \quad \forall i \in N^{h} / i=1, \ldots, n-1 \\
g\left(t_{0}\right)=g\left(t_{n}\right)=0
\end{gathered}
$$


Therefore $g\left(t_{i}\right) \geq 0, \forall i \in N^{h}$ which in turn implies (37).

THEOREM 5.1: The algorithm finishes in a finite number of steps.

Proof: 1. We will prove that if $Y_{\nu, 0}$ is not the solution, then

$$
\exists m / \operatorname{Card}\left(S_{\nu, m}\right)=\operatorname{Card}\left(S_{\nu, 0}\right)-1
$$

In fact, if $Y_{\nu, 0}$ is not the solution, then

- $\operatorname{Card}\left(S_{\nu, 0}\right) \geq 3$

- $\exists$ at least one $i / 1 \leq i \leq n-1 \wedge D^{2}\left(Y_{\nu, 0}, S_{\nu, 0}, i\right)>0$

Let $\hat{\imath}$ be the minimum index with the property (39). While $j_{\nu, m}<\hat{\iota}$, the vector $Y_{\nu, m}$ remains invariant, i.e. $Y_{\nu, m} \equiv Y_{\nu, m-1}$; in consequence, the index $j_{\nu, m}$ will be increasing with $m$; therefore it will exist $\hat{m}$ such that $j_{\nu, \hat{m}}=\hat{\imath}$. Then, we have that:

$$
\begin{gathered}
D^{2}\left(Y_{\nu, \hat{m}-1}, S_{\nu, \hat{m}-1}, \hat{\iota}\right)>0 \\
D^{2}\left(Y_{\nu, \hat{m}-1}, S_{\nu, \hat{m}-1}, \hat{\iota}\right)-Y_{\nu, \hat{m}-1}\left(t_{\hat{\iota}}\right)=D^{2}\left(F, N^{h}, \hat{\iota}\right)-F\left(t_{\hat{\iota}}\right)
\end{gathered}
$$

from (40) we obtain

$$
Y_{\nu, \hat{m}-1}\left(t_{\hat{\imath}}\right) \leq Y L\left(Y_{\nu, \hat{m}-1}, S_{\nu, \hat{m}-1}, \hat{\iota}\right)
$$

From (41) and (42)

$$
-Y L\left(Y_{\nu, \hat{m}-1}, S_{\nu, \hat{m}-1}, \hat{\iota}\right) \leq D^{2}\left(F, N^{h}, \hat{\iota}\right)-F\left(t_{\hat{\iota}}\right)
$$

and by the definition of Step 3 we obtain

$$
S_{\nu, \hat{m}}=S_{\nu, \hat{m}-1}-\{\hat{\imath}\}
$$

which implies (41)

2. By virtue of (42):

$$
\operatorname{Card}\left(S_{\nu+1,0}\right) \leq \operatorname{Card}\left(S_{\nu, m}\right)=\operatorname{Card}\left(S_{\nu, 0}\right)-1
$$

therefore $\nu \leq n=\operatorname{Card}\left(S_{1,0}\right)$, and in consequence the algorithm finishes in a finite number of steps. 


\section{ACCELERATED ALGORITHM OF SHOOTING TYPE}

The two-points boundary value problem which must be solved is the following

$$
Y^{\prime \prime}+\left(Y-F+F^{\prime \prime}\right)^{-}=0 \quad \text { in }(0, T)
$$

with boundary conditions

$$
\begin{gathered}
Y(0)=0 \\
Y(T)=F(T)
\end{gathered}
$$

Equation (43) is transformed into a first-order system of ordinary differential equations

$$
\begin{gathered}
Y^{\prime}=Y D \\
Y D^{\prime}=-\left(Y-F+F^{\prime \prime}\right)^{-}
\end{gathered}
$$

with initial conditions

$$
\begin{gathered}
Y(0)=0 \\
Y D(0)=p
\end{gathered}
$$

The method is based upon solving by discretization the system (46) with initial conditions $Y(0)=0$ and upon adjusting initial condition $Y D(0)$ so as to satisfy the final condition $Y(T)=F(T)$. This search is done with a Newton type algorithm (see $[6,7])$.

\subsection{Equations of the discretized system}

$$
\left.\begin{array}{c}
Y\left(t_{k+1}\right)=Y\left(t_{k}\right)+Y D\left(t_{k}\right) h-\left(F^{\prime \prime}\left(t_{k}\right)-F\left(t_{k}\right)+Y\left(t_{k}\right)\right)^{-} h^{2} \\
Y D\left(t_{k+1}\right)=Y D\left(t_{k}\right)-\left(F^{\prime \prime}\left(t_{k}\right)-F\left(t_{k}\right)+Y\left(t_{k}\right)\right)^{-} h \\
k=1,2,3, \ldots \\
Y\left(t_{1}\right)=Y\left(t_{0}\right)+Y D\left(t_{0}\right) h \\
Y D\left(t_{1}\right)=Y D\left(t_{0}\right)
\end{array}\right\}
$$

Remark 6.1.1: If we denote $p$ the initial slope $Y D(0)$, equations (47) and (48) define an operator

$$
\begin{gathered}
Z: \mathcal{R} \rightarrow \mathcal{R} \\
p \rightarrow Y\left(t_{n}\right)
\end{gathered}
$$


then, the problem consist in finding $p$ such that $Y\left(t_{n}\right)=F(T)$. This nonlinear equation is solved with a Newton method suitably modified so as to be globally convergent.

Remark 6.1.2: It can be easily proved that $Z$ is non-decreasing and piecewise linear, with a finite number of discontinuities of the derivatives. We define operator $Z^{\prime}$ which coincides with the ordinary derivative on the points where it exists, and we extended it to all $\mathcal{R}$ so that it be right continuous. In this way, we have the operator $Z^{\prime}$ necessary for the construction of the following Newton-type algorithm.

\section{Algorithm A2}

Step 0: Choose $p^{0} \in \mathcal{R}, \nu=0$

Step 1: Compute $Z\left(p^{\nu}\right)$

Step 2: If $Z\left(p^{\nu}\right)=F(T)$ then stop

$$
\text { else } p^{\nu+1}=p^{\nu}+\left(Z^{\prime}\left(p^{\nu}\right)\right)^{-1}\left(F(T)-Z\left(p^{\nu}\right)\right)
$$

Step 3: If

$$
\begin{gathered}
\left|F(T)-Z\left(p^{\nu+1}\right)\right|<\left|F(T)-Z\left(p^{\nu}\right)\right| \text { then } \nu=\nu+i \text { and go to Step } 1 \\
\text { Else } p^{\nu+1}=\left(p^{\nu}+p^{\nu+1}\right) / 2 \text { and restart Step } 3 .
\end{gathered}
$$

Remark 6.1.3: Taking into account the previous remarks and the general theory presented in [6], the convergence of this algorithm can be easily obtained.

Remark 6.1.4: In general, algorithm A2 is the most performant one as regards computational time. Nevertheless, for long time intervals, function $Z(p)$ is extremely sensitive with respect to parameter $p$, which implies $Z^{\prime}(p) \approx \infty$ and algorithm A2 may be not convergent due to numerical accuracy problems in Step 2. We can remark, however, that these cases can be solved by applying algorithm $\mathrm{A} 0$ or $\mathrm{A} 1$, which has, in consequence a specific field of application.

\section{APPLICATIONS}

\subsection{The numerical solution for an example with exact solution}

We consider one of the problems given in [2] where:

$$
\begin{gathered}
T=\pi \\
f(t)=\operatorname{sen}(t)
\end{gathered}
$$


Then

$$
F(t)=1-\cos (t)
$$

The numerical solution was calculated by taking a partition of $[0, \pi]$ with $n=100$

The exact solution is

$$
Y(t)=\left\{\begin{array}{lll}
c t & \text { if } & t \in(0, x) \\
c_{1}\left(e^{t}-e^{2 \pi-t}\right)+1-\cos (t) & \text { if } & t \in(\mathrm{x}, \pi)
\end{array}\right.
$$

being

$x=1.753,508 \ldots$ the switching point of the optimal policy $[p=0$ en $(0, x)]$ $c=0.777,523,3 \ldots$

$c_{1}=-0.002,089,587,7 \ldots$

The maximum error between the numerical solution $Y^{h}$ and the exact solution $Y$ is $0.338,9 \mathrm{E}-04$.

7.2. Comparison of the computational time of algorithms A0, A1, A2

$\mathrm{T}_{\text {ABLE }} \mathrm{I}(T=\pi)$

\begin{tabular}{|c|c|c|c|}
\hline$n$ & algorithm A0 & algorithm A1 & algorithm A2 \\
\hline $10 \ldots \ldots \ldots$ & $11.69 \mathrm{sec}$. & $3.07 \mathrm{sec}$. & $3.02 \mathrm{sec}$. \\
$20 \ldots \ldots \ldots$ & 1 min. $07.88 \mathrm{sec}$. & $4.22 \mathrm{sec}$. & $3.78 \mathrm{sec}$. \\
$30 \ldots \ldots \ldots$ & 3 min. $33.55 \mathrm{sec}$ & $5.71 \mathrm{sec}$. & $4.44 \mathrm{sec}$. \\
$40 \ldots \ldots \ldots$ & 7 min. $68.73 \mathrm{sec}$. & $7.57 \mathrm{sec}$. & $5.27 \mathrm{sec}$. \\
\hline
\end{tabular}

TABLE II $(n=40)$

\begin{tabular}{|c|c|c|c|}
\hline$T$ & algorithm A0 & algorithm A1 & algorithm A2 \\
\hline $10 \ldots \ldots \ldots$ & 4 min. 49.34 sec. & $6.26 \mathrm{sec}$. & $6.09 \mathrm{sec}$. \\
$100 \ldots \ldots \ldots$ & 3 min. 28.48 sec. & $4.44 \mathrm{sec}$. & $13.07 \mathrm{sec}$. \\
$150 \ldots \ldots *$ & 3 min. $14.60 \mathrm{sec}$. & $4.66 \mathrm{sec}$. & \\
\hline
\end{tabular}

*** In this case, it was not possible to obtain the final result due to the effects of problems mentioned in remark 6.1.4. 


\section{CONCLUSIONS}

In this paper, following the suggestion mentioned in [2], page 305, we have done an application to the problem of continuous optimal control with quadratic criteria and monotone controls of the methodology stated in [5]. A previous version of these results have been presented in [1]. The method is based upon linear finite elements and discretizations which satisfy a Discrete Maximum Principle (see [3]), and it is an extension of the procedure presented in [4]. The fast algorithm above denoted A1 is based upon the methodology used in [8].

The principle results are the following:

- By virtue of the special regularity $\left(C^{2, \alpha}\right)$ of the exact solution $Y$ it holds an estimate of the discretization error of the type

$$
\left\|Y^{h}-Y\right\| \leq C h^{1 / 2}
$$

- The procedure is globally convergent. Moreover, the convergence is monotone when we choose a discrete supersolution $Y_{0}^{h}$ as initial point for the iterative algorithm.

- Accelerated algorithms have been obtained making use of the simple structure of the system (15)-(18). They are based upon the techniques introduced in [8] and in the methods of shooting type [7].

\section{REFERENCES}

1. L. S. Aragone, Problemas de optimización de sistemas económicos con criterios cuadráticos. Un algoritmo para su solución nunérica, Annales 17 J.A.I.I.O, BuenosAires, 1988, pp. 1076-1088.

2. E. N. Barron, R. JENSEN and A. G. Malliaris, Minimizing a quadratic payoff with monotone controls, Math. Oper. Res., 1987, 12, No. 2.

3. P. G. Ciarlet, Discrete maximum principle for finite-difference operators, Aequationes Math., 1970, 4, No. 3, pp. 338-352.

4. R. L. V. GonzÁlez and E. Rofman, Stochastic controls problems: an algorithm for the value function and optimal policy, Some applications, Proceedings of $13^{\text {th }}$ I.F.I.P. Conference on System Modelling and Optimization, 1987, Tokio, Aug. 31-Sept. 4.

5. R. L. V. González and E. Rofman, On deterministic control problem: an approximation procedure for the optimal cost, Parts I and II, S.I.A.M. J. Control and Opt., 1985, 23, No. 2, pp. 242-266 and 267-285.

6. R. L. V. GonzÁlez and M. M. Tidball, Fast solution of general nonlinear fixed point problems, Rapport de Recherche, No. 1339, I.N.R.I.A., 1990, France. 
7. H. KelleR, Numerical methods for two-point boundary-value problems, Blaisdell Publishing Company, 1968, Massachusetts.

8. M. Medina and R. L. V. González, Fast solution of the obstacle problem, Rapport de Recherche, No. 1241, I.N.R.I.A., 1990, France.

9. J. L. Lions and G. Stampacchi, Variational inequalities, Comm. Pure Applied Math., 1967, 20, 493-519.

10. M. H. Protrer, A first course in real analysis, Springer Verlag, 1977, New York. 